\title{
Propagation Over Plane Earth Through an Exponential Atmosphere
}

\author{
Irvin H. Gerks and Ronald M. Anderson \\ Contribution From the Collins Radio Company, Cedar Rapids, Iowa
}

(Received March 24, 1964; revised May 28, 1964)

\begin{abstract}
An exact solution is found for the field of a dipole over plane, finitely conducting earth through an atmosphere in which the refractivity varies exponentially with height. The method of Hankel transforms is used, so that the final result takes the form of an integral with infinite limits. This integral is evaluated numerically for a typical profile and for several wavelengths, and the results are compared with those for a homogeneous atmosphere. At short wavelengths and large distances, the stratified atmosphere above a plane earth can act as a very efficient waveguide.
\end{abstract}

\section{Introduction}

The solutions of Maxwell's equations for a homogeneous, nonconducting atmosphere over both plane and spherical finitely conducting earth have been known for many years. Almost two decades ago it was revealed by experiments that the field strength at very short wavelengths and large distances is greater than that calculated from "airless-earth" theory by tens of decibels. The favorite theory for explaining this excess is the scattering from randomly located irregularities of refractivity in the air lying within the beams of the two antennas [Booker and Gordon, 1950]. Other authors have maintained that the strong transhorizon fields can be explained only by postulating a prominent contribution from the ever-present stratification [Carroll and Ring, 1955]. An exact solution for a smooth spherical earth and a smooth, continuous profile of refractivity (e.g., exponential) is inordinately difficult. Much work has been done on the basis of solving the wave equation by WKB methods, [Bremmer, 1960], and also by an extension of the latter to the rigorous solution [Bremmer, 1962]. Other authors have employed quasi-optical methods in an effort to allow for the effects of stratification [Bullington, 1963]. A somewhat related problem involving reflection from a stratified ionosphere has been discussed [Wait and Walters, 1963a and 1963b].

It is our opinion that the problem can be formulated in such a manner, especially in the case of "smooth" profiles, that the mathematical difficulties can be overcome with the aid of digital computers. This paper illustrates such a method in the relatively simple case of a plane earth and an exponential profile.

\section{Maxwell's Equations and Boundary Conditions}

A harmonic time function $e^{i \omega t}$ is assumed, and the MKS system of units is employed. Then for a source-free region, Maxwell's equations become

$$
\left.\begin{array}{l}
\operatorname{curl} \mathbf{H}=i \omega \epsilon \mathbf{E} \\
\operatorname{curl} \mathbf{E}=-i \omega \mu \mathbf{H} \\
\operatorname{div} \epsilon \mathbf{E}=0 \\
\operatorname{div} \mathbf{H}=0
\end{array}\right\} \text {. }
$$

Following Bremmer [1949], we assume a vertical electric dipole and express field strength in terms of a scalar Hertz potential $\Pi$ :

$$
\begin{gathered}
\mathbf{E}=\left(k_{0} / k^{2}\right) \operatorname{curl} \operatorname{curl}\left(\mathbf{a}_{z} k \Pi\right), \\
\mathbf{H}=i\left(k_{0} / \omega \mu\right) \operatorname{curl}\left(\mathbf{a}_{z} k \Pi\right),
\end{gathered}
$$

where $\mathbf{a}_{z}$ is a unit vertical vector, $k^{2}=\omega^{2} \mu \epsilon$, and $k_{0}^{2}=\omega^{2} \mu_{0} \epsilon_{0}$. (Zero subscripts designate vacuum values.) Assume $\mu=\mu_{0}, \epsilon=\epsilon(z)$. Substitution of (2) and (3) into (1) yields the scalar wave equation which must be satisfied:

$$
\nabla^{2} \Pi+k^{* 2} \Pi=0
$$

where

$$
*^{*}=k^{2}-k \frac{d^{2}}{d z^{2}}\left(\frac{1}{k}\right)
$$


It is readily shown that, for a smooth profile of tropospheric refractivity, the second term in the right-hand member of (5) is negligible for frequencies greater than about $50 \mathrm{kc} / \mathrm{s}$. For simplicity, we assume $k^{*}=k$.

For an elementary electric dipole located at the point $z=h, r=0, \quad$ (cylindrical coordinates), becomes valid everywhere if we write

$$
\nabla^{2} \Pi+k^{2} \Pi=S,
$$

where

$$
\begin{aligned}
& S=\frac{C}{2 \pi r} \delta(r) \delta(z-h), \\
& C=i \frac{I d l}{\omega \epsilon_{0}} .
\end{aligned}
$$

The solutions are subject to the boundary conditions that the tangential components of $E$ and $H$ must be continuous at the boundary between air and earth, and that the behavior at great height is that characterizing free-space propagation. When a refractivity profile is chosen with all derivatives continuous, the latter requirement will be satisfied by proper choice of the solutions of the differential equation in $z$. The former requirement leads to the equations

$$
\left.\begin{array}{l}
E_{r 1}=E_{r 2} \\
H_{\phi 1}=H_{\phi 2}
\end{array}\right\}(z=0)
$$

where 1 refers to air and 2 to earth. The components of $E$ and $H$ in terms of $\Pi$ are found from (2) and (3). Specifically

$$
\left.\begin{array}{l}
E_{r}=\frac{k_{0}}{k^{2}} \frac{\partial^{2}(k \Pi)}{\partial r \partial z} \\
H_{\phi}=-i \frac{k_{0}}{\omega \mu} \frac{\partial(k \Pi)}{\partial r}
\end{array}\right\} .
$$

Therefore, the boundary conditions specified by (7) require that

$$
\left.\begin{array}{l}
\frac{1}{k_{1}^{2}} \frac{\partial\left(k_{1} \Pi_{1}\right)}{\partial z}=\frac{1}{k_{2}^{2}} \frac{\partial\left(k_{2} \Pi_{2}\right)}{\partial z} \\
k_{1} \Pi_{1}=k_{2} \Pi_{2}
\end{array}\right\}(z=-0) .
$$

It is necessary to find a solution of (6) which also satisfies (9) and the radiation condition at infinity. This is a unique solution.

\section{Solution of the Wave Equation}

In this paper, we assume a smooth exponential profile of refractivity such that

$$
k^{2}(z)=k_{0}^{2}\left(1+a e^{-z / H}\right),
$$

1 More precisely, the wave equation is

$$
\left(\nabla^{2}+k^{* 2}\right) \Pi=i \frac{I d l}{2 \pi r \omega \sqrt{\epsilon_{0} \epsilon}} \delta(r) \delta(z-h) .
$$

where $a=2 N_{s} \times 10^{-6}$ and $H$ is the scale height. We first find appropriate independent solutions of the homogeneous equation

$$
\left(\nabla^{2}+k^{2}\right) \Pi=0 .
$$

The separation of variables is achieved by application of the Hankel transform [Sneddon, 1951]

$$
\tilde{u}(\rho, z)=\int_{0}^{\infty} u(r, z) J_{0}(\rho r) r d r .
$$

This leads to an ordinary differential equation in $z$, which can be solved by any available method. Having found $\tilde{u}(\rho, z)$, we can determine the original function by applying the inverse transform.

$$
u(r, z)=\int_{0}^{\infty} \tilde{u}(\rho, z) J_{0}(\rho r) \rho d \rho .
$$

To apply (12) to the wave equation (11), we multiply by $r J_{0}(\rho r)$ and integrate the result from 0 to $\infty$. The procedure requires integration by parts, recognition of the fact that the functions $r J_{0}(\rho r) \frac{\partial u}{\partial r}$ and $\rho r J_{0}^{\prime}(\rho r) u$ must vanish at $r=0$ and $r=\infty$, and use of the Bessel equation of zero order. The result is, for $u=\Pi$,

$$
\frac{d^{2} \tilde{\mathbf{I}}}{d z^{2}}+\left(k^{2}-\rho^{2}\right) \tilde{\Pi}=0 .
$$

It may be noted that the parameter $\rho$ can be loosely identified with $k \sin \theta$, where $\theta$ is the angle between the wave normal and $z$-axis. The condition $\rho^{2}>k^{2}$ corresponds to the ray-theory concept of total internal reflection. The solutions of (14) should have the nature of progressive waves when $\rho^{2}<k^{2}$ and of standing waves when $\rho^{2}>k^{2}$. It is easy to derive Sommerfeld's solution for a homogeneous atmosphere from (14) and (13) by assuming $k$ constant.

When the exponential profile (10) is assumed, (14) becomes

$$
\frac{d^{2} \tilde{\Pi}}{d z^{2}}+\left(A^{2}+B^{2} e^{-z / H}\right) \tilde{\Pi}=0,
$$

where

$$
\begin{gathered}
A^{2}=k_{0}^{2}-\rho^{2}, \\
B^{2}=k_{0}^{2} a .
\end{gathered}
$$

By the change of variable $v=e^{-z / 2 H},(15)$ is converted to Bessel's equation of imaginary order. We select the solutions

$$
\begin{aligned}
& f(\rho, z)=J_{\nu}(\zeta), \\
& g(\rho, z)=Y_{\nu}(\zeta),
\end{aligned}
$$

where $\nu=i 2 H A, \zeta=2 H B e^{-z / 2 H}$. It can be shown readily that only $f$ has the proper behavior as $z \rightarrow \infty$. A solution of this type was first discussed by Elias (1931).

Consideration of the branch point at $\rho=k_{0}$ leads to the conclusion that, on the appropriate Riemann 
sheet [Brekhovskikh, 1960],

$$
\nu=2 H \sqrt{\rho^{2}-k_{0}^{2}}, \quad \rho^{2}>k_{0}^{2} .
$$

A branch point at $\rho=k_{2}=n k_{0}$ produces no difficulties. Note that we define

$$
n^{2}=\left(k_{2} / k_{0}\right)^{2}=\epsilon_{r}-i 60 \sigma \lambda_{0},
$$

where $\epsilon_{r}$ and $\sigma$ are the dielectric constant and conductivity of the earth.

When the Hankel transform (12) is applied to the source function $S$, we obtain

$$
\tilde{S}=\frac{C}{2 \pi} \delta(z-h)
$$

Since the boundary conditions (9) do not contain $r$ explicitly, they become simply

$$
\left.\begin{array}{l}
\frac{1}{k_{1}^{2}} \frac{d}{d z}\left(k_{1} \tilde{\Pi}_{1}\right)=\frac{1}{k_{2}^{2}} \frac{d}{d z}\left(k_{2} \tilde{\Pi}_{2}\right) \\
k_{1} \tilde{\Pi}_{1}=k_{2} \tilde{\Pi}_{2}
\end{array}\right\} \quad(z=0) .
$$

If the earth is assumed homogeneous, (14) yields the solution

where

$$
\begin{gathered}
\tilde{\pi}_{2}=e^{i \gamma z}, \\
\gamma^{2}=k_{2}^{2}-\rho^{2}=n^{2} k_{0}^{2}-\rho^{2} .
\end{gathered}
$$

Then $\frac{d \tilde{\Pi}_{2}}{d z}=i \gamma \tilde{\Pi}_{2}$, and equations (22) reduce to the single equation

$$
\frac{1}{k_{1}^{2}} \frac{d}{d z}\left(k_{1} \tilde{\Pi}_{1}\right)=\frac{i \gamma}{k_{2}^{2}} k_{1} \tilde{\Pi}_{1} \quad(z=0) .
$$

We can drop the subscript on the symbol Ĩ and write

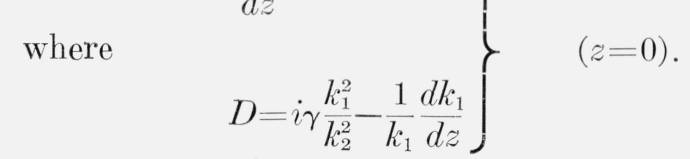

Setting $k_{1}=k_{0}$ except in the derivative and using (10), we get

$$
D=i \frac{\gamma}{n^{2}}+\frac{a}{2 H}
$$

The second term in the right-hand member can be neglected except for very long wavelengths. Finally, it is found that the integration with respect to $\rho$ suggested by (13) yields results only for $\rho$ very close to $k_{0}$, so that we can employ the following condition with negligible error:

$$
D=\frac{i k_{0} \sqrt{n^{2}-1}}{n^{2}} .
$$

This approximation is equivalent to the introduction of a surface impedance, [Wait, 1962].

We can now combine the results given by (18), (19), (21), and (25) by using the Lagrange method of variation of parameters [Ince, 1944]:

$$
\begin{aligned}
\tilde{\Pi}(z)=\frac{1}{W} & {\left[f(z) \int_{0}^{z} g\left(z^{\prime}\right) \tilde{S}\left(z^{\prime}\right) d z^{\prime}+g(z) \int_{z}^{\infty} f\left(z^{\prime}\right) \tilde{S}\left(z^{\prime}\right) d z^{\prime}\right.} \\
& \left.-\frac{D g(0)-g^{\prime}(0)}{D f(0)-f^{\prime}(0)} f(z) \int_{0}^{\infty} f\left(z^{\prime}\right) \tilde{S}\left(z^{\prime}\right) d z^{\prime}\right], \quad \text { (28) }
\end{aligned}
$$

where $z^{\prime}$ is a variable of integration and the Wronskian $W$ is given by

$$
W[f(z), g(z)]=-\frac{1}{\pi H} .
$$

Evaluation of the integrals in (28) gives different results, depending upon whether $z>h$ or $z<h$. For $z>h$,

$$
\tilde{\Pi}(\rho, z)=-\frac{C H}{2} f(z)\left[g(h)-\frac{D g(0)-g^{\prime}(0)}{D f(0)-f^{\prime}(0)} f(h)\right] .
$$

For $z<h, z$ and $h$ are reversed. Finally substitution in (13) yields the solution, for $z>h$,

$$
\begin{aligned}
\Pi(r, z)=- & \frac{C H}{2} \int_{0}^{\infty} f(z) \\
& {\left[g(h)-\frac{D g(0)-g^{\prime}(0)}{D f(0)-f^{\prime}(0)} f(h)\right] J_{0}(\rho r) \rho d \rho . }
\end{aligned}
$$

It is understood that $f$ and $g$ are functions of $\rho$ as well as $z$. Since $\rho$ appears in the expression for $\nu$, the order of the Bessel functions in (18) and (19), it is seen that this order ranges from large imaginary values to zero, then through positive real values to infinity as $\rho$ goes from 0 to $\infty$ along the real $\rho$-axis. The integration with respect to $\rho$ can be thought of as an addition of rays propagated at various angles relative to the $z$-axis. Small values of $\rho$ correspond to rays which escape from the atmosphere. The condition $\rho=k_{\mathrm{c}}$ corresponds to the onset of total internal reflection at a great height. As $\rho$ continues to increase, the rays are reflected at lower elevations.

It may also be noted that the integrand in (31) develops poles at values of $\rho$ where $D f(0)=f^{\prime}(0)$. These poles lie on the positive real $\rho$ axis when $D$ is real or zero and slightly below this axis when $D$ is complex. These poles are associated with the physical concept of multiple reflection of rays between the atmosphere and the earth. For long wavelengths there may be but a single pole, whereas for short wavelengths there may be many poles.

No serious effort has been made to evaluate the integral in (31) by such familiar means as the saddlepoint method. Much better control over the accuracy of the results can be achieved by numerical integration. Also, it is not considered very important at this stage to investigate the effect of antenna height variations. Of much more interest is the 
variation of field strength with horizontal distance. Accordingly, we let $z=h=0$, and obtain the relatively simple result

$$
\begin{aligned}
\Pi(r, 0) & =-\frac{C H}{2} \int_{0}^{\infty} f(0) \frac{f(0) g^{\prime}(0)-f^{\prime}(0) g(0)}{D f(0)-f^{\prime}(0)} J_{0}(\rho r) \rho d \rho \\
& =\frac{C}{2 \pi} \int_{0}^{\infty} \frac{J_{0}(\rho r) \rho d \rho}{D-f^{\prime}(0) / f(0)} .
\end{aligned}
$$

We note that

$$
\begin{array}{rlrl}
f^{\prime}(0) / f(0) & =-B J_{\nu}^{\prime}(2 H B) / J_{\nu}(2 H B), \\
\nu & =i 2 H \sqrt{k_{0}^{2}-\rho^{2}} & \rho<k_{0} \\
& =2 H \sqrt{\rho^{2}-k_{0}^{2}} & \rho>k_{0} .
\end{array}
$$

It is interesting to note that

$$
\lim _{\rho \rightarrow \infty}\left[f^{\prime}(0) / f(0)\right]=-B \frac{\nu}{2 H B}=-\sqrt{\rho^{2}-k_{0}^{2}},
$$

and that the limiting form of (32) is the familiar Sommerfeld solution for a homogeneous atmosphere. Since $2 H k_{0}$ is normally a large number,

$$
\lim _{\rho \rightarrow 0}\left[f^{\prime}(0) / f(0)\right] \approx-i \sqrt{k_{0}^{2}-\rho^{2}},
$$

and a similar coincidence with the Sommerfeld solution results. In fact, for a homogeneous medium, (32) takes the form

$$
\mathrm{II}(r, 0)=\frac{C}{2 \pi} \int_{0}^{\infty} \frac{J_{0}(\rho r) \rho d \rho}{D+\nu / 2 H} .
$$

Dimensionless forms can be obtained if we let $\rho=k_{0} v$. Equation (32) now becomes

$$
\Pi(r, 0)=\frac{C k_{0}}{2 \pi} \int_{0}^{\infty} \frac{J_{0}\left(k_{0} r v\right) v d v}{\left(D / k_{0}\right)+\sqrt{a} J_{\nu}^{\prime}(2 H B) / J_{\nu}(2 H B)},
$$

where

$$
\begin{aligned}
\nu & =i 2 H k_{0} \sqrt{1-v^{2}} \quad v<1 \\
& =2 H k_{0} \sqrt{v^{2}-1} \quad v>1 .
\end{aligned}
$$

A corresponding form is obtained for (33). Now only the coefficient, $C k_{0}$, has dimensions. If the result obtained from (32) or (33) is divided by the free-space value

$$
\Pi_{0}=-\frac{C}{4 \pi r} e^{-i k_{0} r},
$$

a dimensionless ratio is obtained, which can be plotted as a function of $r$. The shape of such a curve is essentially the same as if the vertical component of $E$ had been computed and compared with its free-space value.
It is often convenient to use complex variable theory to evaluate the integral. It is first necessary to extend the path of integration along the entire real axis by a familiar transformation of the integrand [Sommerfeld, 1949]. Equation (32a) takes the form

$$
\Pi(r, 0)=\frac{C k_{0}}{4 \pi} \int_{-\infty}^{\infty} \frac{H_{0}^{(2)}\left(k_{0} r v\right) v d v}{\left(D / k_{0}\right)+\sqrt{a} J_{\nu}^{\prime}(2 H B) / J_{\nu}(2 H B)} .
$$

If we divide (35) by (34), we get finally

$$
\frac{\Pi}{\Pi_{0}}=-k_{0} r e^{i k_{0} r} \int_{-\infty}^{\infty} \frac{H_{0}^{(2)}\left(k_{0} r v\right) v d v}{\left(D / k_{0}\right)+\sqrt{a} J_{\nu}^{\prime}(2 H B) / J_{\nu}(2 H B)} .
$$

One method of evaluating the integral is to choose a path along the real axis. Then we use (32a), employ an asymptotic form for $\boldsymbol{J}_{0}$ similar to (38), let $r$ be an integral number of wavelengths, and obtain

$$
\frac{\Pi}{\Pi_{0}} \approx-2 \sqrt{\frac{2 k_{0}}{\pi}} \int_{0}^{\infty} \frac{\cos \left(k_{0} r v-\pi / 4\right) v d v}{\left(D / k_{0}\right)+\sqrt{a} J_{\nu}^{\prime}(2 H B) / J_{\nu}(2 H B)} .
$$

Since the cosine term varies rapidly with $v$ when $k_{0} r$ becomes large, it is very helpful to choose a step size $\nabla v$ such that all of the integrand except the cosine term can be accurately represented by a second-degree polynomial, then integrate analytically. In this way, the cosine term may execute many cycles within one step without degrading the accuracy of the computed value of the integral.

A second method is based upon Cauchy's integral theorem. The integrand in (36) is an analytic function of $v$ except at the poles, which are located in the second and fourth quadrants of the complex $v$ plane. The path of integration is distorted into the lower half-plane (fig. 1), where the integrand vanishes sufficiently far off the real axis. However, the path must be taken around the branch points at $v=1$ and $v=n$. The contribution from the second branch point is negligible for normal values of $n$. The cut from the branch point at $v=1$ is drawn downward, and the path of integration is collapsed onto opposite banks of this cut. The total value of the integral is the sum of this branchcut integral and of the sum of the residues multiplied by $2 \pi i$.

Let $\Pi_{1}$ be the contribution from the branch point at $v=1$, and let $v=1-i \delta$ along this part of the path. The integrand on the two banks of the cut differs only in that $\nu$, the order of the Bessel function, changes sign. In the definition $\nu=i 2 H k_{0} \sqrt{1-v^{2}}=$ $i 2 H k_{0} \sqrt{\delta^{2}+i 2 \delta}$, the radical must be taken with a positive sign on the left bank and a negative sign on the right bank of the cut. For simplicity in writing a formula, we always take the positive sign and change $\nu$ to $-\nu$ on the right bank. Now, if we take advantage of the fact that the significant range of $v$ is in the vicinity of 1 and decide to keep the minimum value of $r$ about 10 wavelengths, we can use a simple asymptotic form for the Hankel 


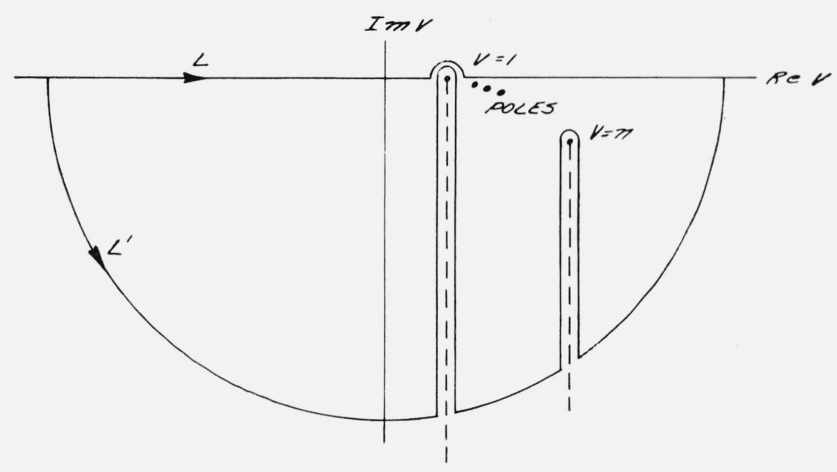

Figure 1. Path of integration in complex v-plane for (36).

function:

$$
\begin{aligned}
H_{0}^{(2)}\left(k_{0} r v\right) & \approx \sqrt{\frac{2}{\pi k_{0} r}} e^{-i\left(k_{0} r v-\pi / 4\right)} \\
& =\sqrt{\frac{2}{\pi k_{0} r}} e^{-i\left(k_{0} r-\pi / 4\right)} e^{i k_{0} r(1-v)} .
\end{aligned}
$$

Equation (36) leads to the form

$$
\frac{\Pi_{1}}{\Pi_{0}} \approx i \sqrt{\frac{2 k_{0} r}{\pi}} e^{i \pi / 4} \int_{0}^{\infty} e^{-k_{0} r \delta}[] d \delta
$$

where the brackets are to be filled by the expression

$$
\begin{aligned}
\frac{1}{\left(D / k_{0}\right)+\sqrt{a} J_{-\nu}^{\prime}(2 H B) / J_{-\nu}(2 H B)} & \\
& -\frac{1}{\left(D / k_{0}\right)+\sqrt{a} J_{\nu}^{\prime}(2 H B) / J_{\nu}(2 H B)}
\end{aligned} .
$$

This integral converges rapidly when $k_{0} r$ is large.

The location of a pole may be defined by the following relation which corresponds to a zero of the denominator of the integrand:

where

$$
J_{\nu_{s}}(2 H B)=\tau J_{\nu_{s}}^{\prime}(2 H B) \text {, }
$$

$$
\tau=i \frac{\sqrt{a n^{2}}}{\sqrt{n^{2}-1}}
$$

Since $\tau$ is normally small, the poles are located just below the positive real $v$ axis, and near the real-axis zeros of $J_{\nu}(2 H B)$. Furthermore, the realaxis zeros must lie in the range $0<\nu<2 H B$, i.e., in the range where $v$ is slightly greater than 1 . Equation (40) may be solved for $\nu_{s}$ by an iterative method. Then

$$
v_{s}=\sqrt{1+\nu_{s}^{2} / 4 H^{2} k_{0}^{2}}
$$

Let $\Pi_{2}$ be the contribution of the poles. This is made up of $2 \pi i$ times the residues at these poles. The residue at a pole is obtained by differentiating the denominator of (36) with respect to $v$, then substituting $v=v_{s}$. We obtain

$$
\begin{aligned}
& \frac{d}{d v}(\text { denominator })=\frac{\left(2 H k_{0}\right)^{2} \sqrt{a} v}{\nu} \frac{d}{d \nu}\left[J_{\nu}^{\prime}(2 H B) / J_{\nu}(2 H B)\right] \\
& =-\frac{\left(2 H k_{0}\right)^{2} \sqrt{a} v}{\nu \tau^{2}} \frac{d}{d \nu} \\
& {\left[J_{\nu}(2 H B) / J_{\nu}^{\prime}(2 H B)\right] \text {, }} \\
& 1-v_{s}=\frac{-v_{s}^{2}}{\left(2 H k_{0}\right)^{2}\left(1+v_{s}\right)} \approx-\frac{v_{s}^{2}}{2\left(2 H k_{0}\right)^{2}}, \\
& \frac{\Pi_{2}}{\Pi_{0}} \approx-i \frac{2 \pi \tau^{2}}{\left(2 H k_{0}\right)^{2} \sqrt{a}} \sqrt{\frac{2 k_{0} r}{\pi}} e^{i \pi / 4} \\
& \times \sum_{s=1} \frac{\nu_{s} \exp \left[-i k_{0} r \nu_{s}^{2} / 2\left(2 H k_{0}\right)^{2}\right]}{\frac{d}{d \nu}\left[J_{\nu}(2 H B) / J_{\nu}^{\prime}(2 H B)\right]_{v=v_{s}}} .
\end{aligned}
$$

Since $\nu_{s}$ normally has only a small negative imaginary component, the damping factor for the residues tends to remain near unity, even for large values of $k_{0} r$. This means small attenuation of the trapped modes.

The total Hertz potential relative to its free-space value is the sum of (39) and (43). For long wavelengths, where $2 H B$ is small, there may be only one root of eq (40), therefore only one pole. For short wavelengths, there are many roots of (40) and many poles. This behavior is somewhat similar to that of a metallic wave-guide, in which many modes can propagate at sufficiently short wavelengths.

\section{Computations}

Only a few comments are required with regard to the computation of numerical results. The evaluation of the integral in (37) or (39) by an application of Simpson's method is routine except for the rapid oscillation of the cosine function in (37). This is a common problem in Fourier analysis. It is generally somewhat easier to use complex-plane integration, especially when $2 H B$ is large and $J_{\nu}(2 H B)$ has many zeros on the real $v$-axis.

Perhaps no other functions have been so extensively investigated as the solutions of Bessel's equation. The work of Langer [1931] and Watson [1944] is especially noteworthy. Since both the argument and order of the Bessel function are often large in this application, and since the order may be complex, asymptotic formulas must be used and care must be exercised in choosing the formula which is appropriate to the particular region of the complex plane (Stokes phenomenon). It is also to be noted that computation tends to be difficult in the region where the order and argument of the Bessel function become equal, i.e., the region where the coefficient of $\tilde{I}$ in (15) becomes zero. In the present problem, this difficulty occurs only when integration along the real axis is employed, since the argument of the Bessel function is always real. It can be avoided in the simple case of zero antenna heights, because a 
familiar recursion formula can be used for computing $J^{\prime} / J$. A useful form is the following:

$$
\frac{J_{\nu}^{\prime}(\zeta)}{J_{\nu}(\zeta)}=\frac{\nu}{\zeta}-\frac{1}{\frac{\nu+1}{\zeta}+\frac{J_{\nu+1}^{\prime}(\zeta)}{J_{\nu+1}(\zeta)}}
$$

The ratio $J^{\prime} / J$ is first computed for a larger order $\nu+n$, where $n$ is an integer, from a simple asymptotic formula, and an iterative procedure is used to reach the final result. The location of the poles $\nu_{s}$ in accordance with (40) also presents some difficulties. The general procedure is to obtain a first estimate of $\nu_{s}$ and then make corrections by an iterative method until (40) is satisfied. Two cases may be distinguished, viz, $2 H B$ small and $2 H B$ large. In the former case a first estimate of $\nu_{1}$ can be obtained from the formula

$$
\frac{1}{\tau}=\frac{J_{\nu_{1}}^{\prime}(2 H B)}{J_{\nu_{1}}(2 H B)} \approx \frac{\nu_{1}}{2 H B}-\frac{H B}{\nu_{1}+1} .
$$

In the latter case, numerous poles exist, so that $\left|\nu_{1}\right|<<2 H B$, and we can use a simple asymptotic formula for a first estimate:

$$
\frac{1}{\tau}=\frac{J_{\nu_{1}}^{\prime}(2 H B)}{J_{\nu_{1}}(2 H B)} \approx-\tan \left(2 H B-\frac{\pi \nu_{1}}{2}-\frac{\pi}{4}\right) .
$$

The iterative method for improving upon such an estimate is based upon the numerical determination of the derivative of $J / J^{\prime}$ with respect to $\nu$. In this process, the derivative appearing in (43) is also computed. Where several poles occur, extrapolation methods can be used to good advantage for a first estimate after the pole of lowest order is located.

In the following numerical examples, both realaxis and complex-plane integration have been employed, and the results were found to agree.

\section{Numerical Examples}

(a) $f=100 \mathrm{kc} / \mathrm{s}, \lambda_{0}=3000 \mathrm{~m}, \epsilon_{r}=10, \sigma=10^{-2} \mathrm{mho} / \mathrm{m}$, $n^{2}=10-i 1800, a=6 \times 10^{-4}, H=6000 \mathrm{~m}, 2 H K_{0}=8 \pi=$ $25.1327,2 H B=8 \pi \sqrt{6 \times 10^{-4}}=0.615624$.

Vertical electric dipole on the surface:

$$
\tau=1.03924 \exp i 0.78846, \nu=i 25.1327 \sqrt{1-v^{2}} .
$$

Location of pole:

first estimate from $(45) \ldots \nu_{1} \approx 0.535-i 0.390$, corrected location . . . $\nu_{1}=0.53581-i 0.38902$.

The ratio of the Hertz potential to that in free space was computed with the aid of a large digital computer as a function of horizontal distance from the source. The results are shown in figure 2. The effect of the stratified atmosphere is discernible at long distances but is too small to be important practically.

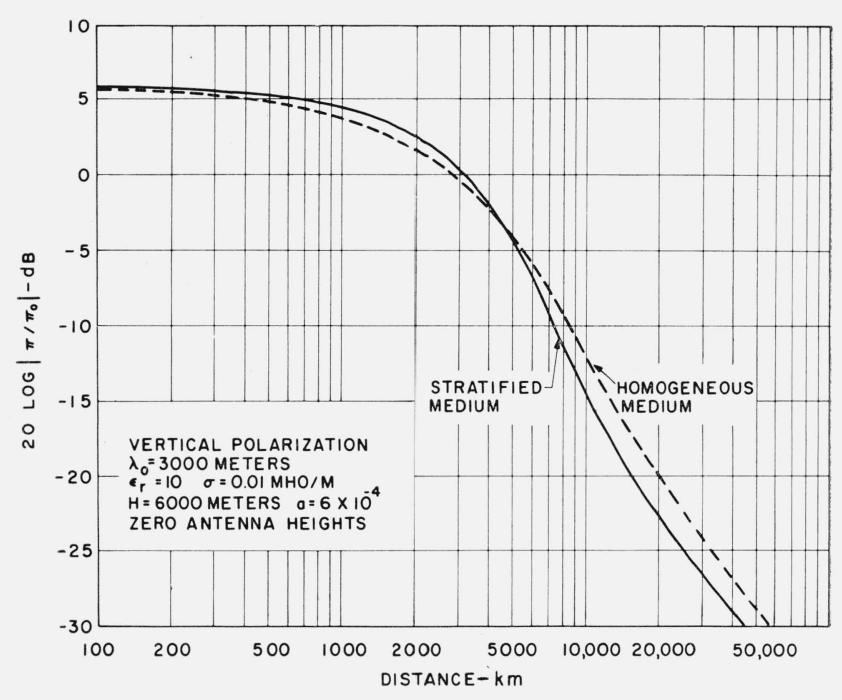

Figure 2. Hertz potential relative to free-space value. $\lambda_{\mathrm{o}}=3000 \mathrm{~m}$.

(b) $f=10 \mathrm{Mc} / \mathrm{s}, \lambda_{\mathrm{o}}=30 \mathrm{~m}, \epsilon_{r}=10, \sigma=10^{-2} \mathrm{mho} / \mathrm{m}$, $n^{2}=10-i 18 . \quad a=6 \times 10^{-4}, \quad H=6000 \quad \mathrm{~m}, \quad 2 H K_{0}$ $=800 \pi=2513.27,2 H B=800 \pi \sqrt{6 \times 10^{-4}}=61.5624$.

Vertical electric dipole on the surface:

$\tau=0.11243 \exp i 1.06065, \nu=i 2513.27 \sqrt{1-v^{2}}$.

Location of poles:

first estimate from $(46)-\nu_{1} \approx 1.6565-i 0.06247$, corrected location $-\nu_{1}=1.6697-i 0.063495$.

$$
\begin{array}{ll}
\nu_{2}=3.7271-i 0.064808 & \nu_{11}=24.9130-i 0.077705 \\
\nu_{3}=5.8313-i 0.066138 & \nu_{12}=27.6957-i 0.079327 \\
\nu_{4}=7.9857-i 0.067486 & \nu_{13}=30.6126-i 0.081015 \\
\nu_{5}=10.1942-i 0.068856 & \nu_{14}=33.6901-i 0.082779 \\
\nu_{6}=12.4616-i 0.070251 & \nu_{15}=36.9658-i 0.084639 \\
\nu_{7}=14.7932-i 0.071672 & \nu_{16}=40.4966-i 0.086638 \\
\nu_{8}=17.1954-i 0.073123 & \nu_{17}=44.3770-i 0.088816 \\
\nu_{9}=19.6762-i 0.074610 & \nu_{18}=48.7895-i 0.091324 \\
\nu_{10}=22.2447-i 0.076132 & \nu_{19}=54.2070-i 0.094847
\end{array}
$$

The results in the form of relative Hertz potential versus distance are shown in figure 3 . In this case, the effect of the stratified atmosphere is very prominent at long distances. It is apparent that the trapped modes account for a slowly decaying field in this distance range. The fluctuations due to mode interference become so numerous and irregular for distances greater than $1500 \mathrm{~km}$ that only the square root of the sum of the squares of mode amplitudes was plotted. In this region, the amplitude is a very sensitive function of distance or of variations in the parameters of the refractivity profile. Temporal variations of these parameters can be expected to produce fading at a fixed distance. The situation is somewhat similar to that believed to exist in short-wave transhorizon propagation. 


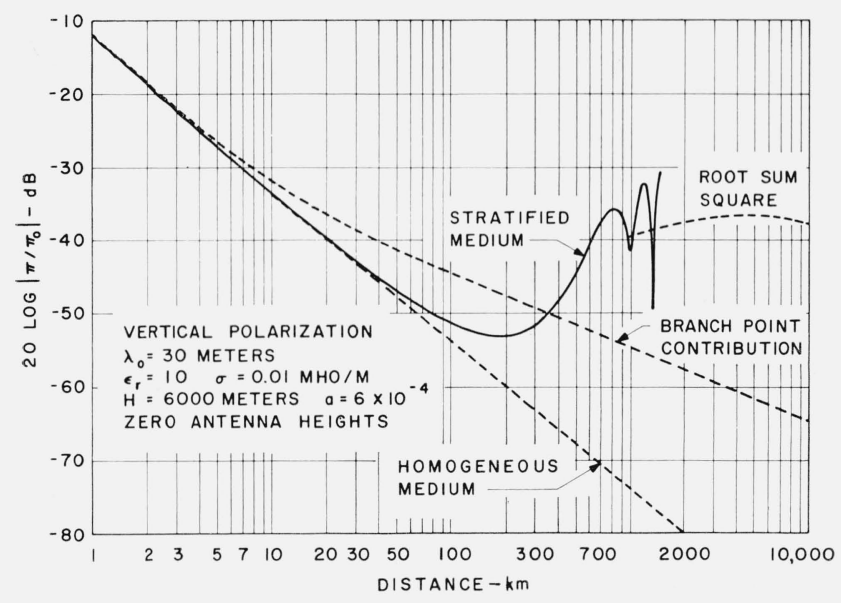

Figure 3. Hertz potential relative to free-space value. $\lambda_{\mathrm{o}}=30 \mathrm{~m}$.

\section{Conclusions}

A practical method has been developed which allows computation of the Hertz potential produced by a vertical dipole over a finitely conducting earth and in an atmosphere with an exponential profile of refractivity. The mathematical solution is an integral, which must be evaluated by numerical methods. The Cauchy integral theorem may be applied to show the existence of one or more trapped modes. For long wavelengths, such as $3000 \mathrm{~m}$, the attenuation of the one trapped mode is rather large, and the influence of the stratification of the atmosphere is slight. In the range of $10,000 \mathrm{~km}$ and beyond in figure 2, the result comes almost entirely from the branch-cut integral (39). For shorter wavelengths, such as $30 \mathrm{~m}$, numerous trapped modes exist and the attenuation as a function of distance is amazingly low. Beyond 600 or $700 \mathrm{~km}$ in figure 3, the field results in large part from the residues (43). At long distances, these residues interfere to produce rapid fluctuations of field strength as a function of distance. This could lead to fading at a fixed distance if the atmospheric structure varied slightly with time. The situation seems somewhat analogous to that encountered in scatter propagation.

This paper deals with a case which is not in keeping with the spherical geometry of the real world.
However, the wave equation can be solved readily for this case, whereas the same is not true in a spherical system. It is believed that certain of the concepts developed here may be useful in an attack on the more difficult problem of propagation in a stratified medium surrounding a sphere.

\section{References}

Booker, H. G. and W. E. Gordon (April 1950), A theory of radio scattering in the troposphere, Proc. IRE 38, 401-412.

Brekhovskikh, L. M. (1960), Waves in Layered Media (Academic Press, Inc., New York, N.Y.).

Bremmer, H. (1949), Terrestrial Radio Waves (Elsevier Publishing Co., New York, N.Y.).

Bremmer, H. (Sept.-Oct. 1960), On the theory of wave propagation through a concentrically stratified troposphere with a smooth profile. Part I. Discussion of the extended WKB approximation, J. Res. NBS 64D (Radio Prop.), No. 5, 467-482.

Bremmer, H. (Jan.-Feb. 1962), On the theory of wave propagation through a concentrically stratified troposphere with a smooth profile. Part II. Expansion of the rigorous solution, J. Res. NBS 66D (Radio Prop.), No 1, 31-52.

Bullington, K. (Nov. 1963), Reflections from an exponential atmosphere, Bell System Tech. J. 43, No. 6, 2849-2867.

Carroll, T. J. and R. M. Ring (Oct. 1955), Propagation of short radio waves in a normally stratified troposphere, Proc. IRE 43, 1384-1390.

Elias, G. J. (1931), Reflection of electromagnetic waves at ionized media with variable conductivity and dielectric constant, Proc. IRE 19, 891-907.

Ince, E. L. (1944), Ordinary Differential Equation (Longmans, Green and Co., Ltd., Dover, New York).

Langer, R. E. (Jan. 1931), On the asymptotic solutions of ordinary differential equations, with an application to the Bessel functions of large order, Trans. Am. Math. Soc. 33, 23.

Sneddon, I. N. (1951), Fourier Transforms (McGraw-Hill Book Co., New York, N.Y.).

Sommerfeld, A. (1949), Partial Differential Equations (Academic Press, Inc., New York, N.Y.).

Wait, J. R. (1962), Electromagnetic Waves in Stratified Media (Pergamon Press, London, England).

Wait, J. R. and L. C. Walters (Sept.-Oct. 1963a), Reflection of VLF radio waves from an inhomogeneous ionosphere. Part II. Perturbed exponential model, J. Res. NBS 6rD (Radio Prop.), No. 5, 519-523.

Wait, J. R. and L. C. Walters (Nov.-Dec. 1963b), Reflection of VLF radio waves from an inhomogeneous ionosphere. Part III. Exponential model with hyperbolic transition, J. Res. NBS 69D (Radio Prop.), No. 6, 747-752.

Watson, G. N. (1944), Theory of Bessel Function, 2d ed. (The Macmillan Co., New York, N.Y.).

(Paper 68D 11-420) 